\title{
Raffaele de Cesare: sul filo della memoria
}

\section{Mario Richter}

\section{(2) OpenEdition}

\section{Journals}

\section{Edizione digitale}

URL: http://journals.openedition.org/studifrancesi/7786

DOI: 10.4000/studifrancesi.7786

ISSN: 2427-5856

\section{Editore}

Rosenberg \& Sellier

\section{Edizione cartacea}

Data di pubblicazione: 1 juillet 2009

Paginazione: 231-233

ISSN: 0039-2944

\section{Notizia bibliografica digitale}

Mario Richter, «Raffaele de Cesare: sul filo della memoria», Studi Francesi [Online], 158 (LIII | II) | 2009, online dal 30 novembre 2015, consultato il 12 janvier 2021. URL: http://journals.openedition.org/ studifrancesi/7786 ; DOI: https://doi.org/10.4000/studifrancesi.7786

\section{(c) (i) (9)}

Studi Francesi è distribuita con Licenza Creative Commons Attribuzione - Non commerciale - Non opere derivate 4.0 Internazionale. 


\section{Raffaele de Cesare: sul filo della memoria}

Conobbi Raffaele de Cesare nella primavera del 1960. Non ho dimenticato le circostanze di quel mio lontano primo incontro con lui. Mi ero da poco laureato a Padova con una tesi sulla poesia di Jean de Sponde. La mia famiglia, dopo una lunga parentesi veneta, era nel frattempo tornata a risiedere a Milano. Per me l'ambiente era pressoché nuovo. Desiderando continuare e approfondire gli studi cominciati a Padova, magari seguendo uno degli allora previsti corsi di specializzazione, un po' guidato dal caso (o da ciò che si ritiene tale), un bel giorno fui portato dai miei passi a varcare la soglia dell'Università Cattolica. Eccomi all'Istituto di Filologia moderna. Sono però ancora incerto in quale disciplina proseguire gli studi: letteratura francese o letteratura italiana? Il mio interesse è infatti vigile per tutt'e due. Passo davanti allo studio di Mario Apollonio, italianista, il cui nome mi è noto. Proprio in quel momento, scorgo il professore mentre esce dallo studio. Mi faccio coraggio: lo fermo, mi presento e gli mostro la mia tesi. Non ho il tempo di aggiungere nemmeno una parola che il professore mi consiglia di andare nello studio in fondo al corridoio a destra. «Là - mi dice in fretta - trova chi fa per lei. È un giovane professore di letteratura francese. Si chiama Raffaele de Cesare. Vedrà, con lui si troverà senz'altro bene». Fu così che senza indugio andai a bussare allo studio di de Cesare. Mi è sempre rimasta chiarissima nella memoria la schietta cordialità con cui il professore mi disse, vedendomi e accogliendomi per la prima volta, «caro amico!». Poteva certo trattarsi di una formula banale, ma, pronunciata da lui, decisamente non lo era.

Si mostrò subito interessato al mio lavoro, alla mia provenienza, ai miei interessi. Mentre parlava, ebbi modo di notare la sua straordinaria capacità di instaurare un rapporto semplice e naturale, senza che ciò venisse comunque a intaccare un certo suo professorale, autorevole, ma pur sempre affabile distacco. Era..., sì, «bello e di gentile aspetto». Mi promise che avrebbe esaminato con attenzione la mia tesi di laurea e mi disse che mi facessi rivedere di lì a un mese. In realtà, un intero anno passò prima che potessi rifarmi vivo con lui. Si era infatti per me interposta l'improvvisa chiamata al servizio militare come allievo ufficiale. Approfittando di una breve licenza, mi ripresentai un giorno in smagliante divisa nello studio di de Cesare. Quella mia nuova immagine lo sorprese e visibilmente gli piacque, anche perché - come disse - gli ricordava una sua analoga precedente esperienza, sebbene quella fosse stata da lui vissuta in circostanze ben più drammatiche sul fronte francese dell'ultima sciagurata guerra mondiale. Capii subito che quella mia ormai inattesa riapparizione gli risultava particolarmente gradita. Da tempo aveva letto con interesse la mia tesi, tanto che adesso mi proponeva di ricavarne un articolo per “Ævum” (la rivista della Cattolica). Mi consigliava anche di riprendere al più presto gli studi e di non «perdere tempo» (parole sue) con un corso di perfezionamento. Si adoperò invece per cercarmi un'attività che mi consentisse di compiere nelle più favorevoli condizioni le mie ricerche. A questo scopo scrisse al suo collega Franco Simone (dell'Università di Torino, fondatore e direttore della già prestigiosa rivista "Studi Francesi") perché appoggiasse una mia candidatura a un posto di lettore d'italiano presso l'Università 
di Grenoble. Di lì a poco mi fece nominare suo assistente (volontario, s'intende, come erano allora tutti quelli della Cattolica) chiedendomi soltanto di fargli qualche volta compagnia agli esami. Ben presto, con mia somma meraviglia, mi disse di presentarmi alla «libera docenza», a cui egli attribuiva una particolare importanza. Fu insomma estremamente generoso. Mi aiutava, m'incoraggiava, mi dava saggi consigli, soprattutto leggeva con la più esigente accuratezza ogni lavoro che via via sottomettevo al suo giudizio. Alla sua attività, ai suoi personali progetti di ricerca (che erano molti) non faceva mai il minimo cenno. Ciò è provato dal fatto che, volendo parlare di lui, mi vedo continuamente costretto a parlare di me. Rispettava pienamente l'orientamento delle mie curiosità, dei miei gusti, dei miei metodi, degli autori che più in quel momento mi attiravano e che erano molto distanti (come, ad esempio, Duplessis-Mornay) da quelli di cui lui si occupava con abituale superlativo rigore, soprattutto, come si sapeva, in vista di una sempre più approfondita conoscenza della presenza in Italia del suo prediletto Balzac. Soltanto una volta, quasi scusandosi, disse che avrebbe gradito una mia ricerca di tipo propriamente storico. Non tardai a portargli un primo capitolo riguardante l'attività di Ardengo Soffici nella Parigi del primo Novecento. Si dichiarò molto soddisfatto di quel mio lavoro di natura documentaria e sembrava esserne persino interessato, anche se io capivo che dovevo alla sua singolare liberalità di studioso, alla sua delicata comprensione delle altrui curiosità l'accettazione di quell'autore. Ho così sempre tenuto sopra ogni cosa a diventare, in questo, il suo più devoto allievo, pur essendo fin da allora consapevole che mai sarei riuscito nemmeno lontanamente ad avvicinarmi a lui in tanto raro magistero. A poco a poco si accese fra noi una corrente di amicizia, quella che deve comunque conciliarsi col naturale rispetto che l'allievo deve al maestro.

Una domenica pomeriggio, il professor de Cesare mi chiamò al telefono: «Caro Richter, che ne pensa di fare un giretto al parco di Monza?». Lusingatissimo e non meno stupitissimo della proposta, fui pronto a farmi trovare all'appuntamento stabilito. Passeggiammo a lungo negli squadrati viali dell'ampio parco. Era una tiepida giornata d'autunno. In quella circostanza, de Cesare mi parlò anche un po' di sé, si lasciò sfuggire qualche confidenza, ma sempre con la sorridente e quasi ironica riservatezza che era l'abito stesso della sua personalità. E poi io ero a mia volta troppo rispettosamente esitante per tentare di forzare in qualche modo quel suo chiuso mondo di segreti inespressi. Erano giorni in cui il caro professore avvertiva con maggiore peso la solitudine in cui egli, privo di famiglia, viveva a Milano.

Col tempo i nostri rapporti si fecero sempre più frequenti, sempre più aperti e distesi, fino al momento in cui, arrivando io (soprattutto grazie a lui) alla cattedra, mi accordò il «tu» dei colleghi. Ma la formalità accademica non fu altro che la naturale ratifica di una familiarità che ormai ci legava da tempo.

Nel corso della mia vita ho sempre cercato di essere il più possibile degno dell'esempio datomi da de Cesare. Ho conosciuto in lui il perfetto galantuomo, lo studioso vero, umile ed esigentissimo nella verifica dei documenti, del tutto estraneo ad ogni forma di esibizione. Non conosceva compromessi nell'affermazione di ciò che riteneva giusto. Più di una volta lo vidi persino adirarsi di fronte agli arroganti soprusi che si rivelassero apertamente lesivi dei diritti da lui attribuiti allo «Spirito» (così si esprimeva). Naturalmente questo suo adamantino e inflessibile comportamento finì con l'assicurargli un certo isolamento, del quale mai si lamentò e che anzi egli coltivò gelosamente per proseguire fino all'ultimo le sue amatissime e preziose ricerche. Senza dubbio de Cesare è stato, soprattutto sul piano dell'indagine storica, uno dei più solidi e seri e attendibili francesisti del nostro tempo.

Tornando di recente a leggere un suo libro (credo l'ultimo) amorevolmente dedicato alle lettere che gli furono nel tempo inviate dall'illustre professore di letteratura latina medievale e rettore della Cattolica Ezio Franceschini (EzIo FrAnCESCHINI, Let- 
tere a Raffaele de Cesare, raccolte, trascritte, annotate a cura del destinatario, Milano, Vita e Pensiero, 2006), ho avvertito vivo il desiderio di fargli sapere per lettera che «libri così fanno bene al cuore, perché sono spaccati di verità umana, con le sue luci, con le sue ombre». La risposta di de Cesare, oltre ad essere l'ultima sua lettera a me spedita, mi sembra riassumere in modo esemplare la sua più intima natura di uomo umile, generoso, disinteressato, sinceramente innamorato degli studi. Mi concedo la libertà di trascriverla qui testualmente:

Mio carissimo Mario,

Città di Castello, 4 ott. 2008

Jeri ho ricevuto la tua affettuosa lettera a cui rispondo a giro di posta con i più vivi ringraziamenti.

Ogni volta che tu mi scrivi, mi arricchisci di una gioja intensa. Dalla tua cultura ho molto appreso; nel tuo animo ho conosciuto il calore di una amicizia che in questi miei ultimi anni è ancora più vivida che non nei giorni - ormai lontani - in cui ci si ritrovava a Milano. E, poi, tu sei così generoso nel giudizio delle non molte - e sempre imperfette - cose che ho scritte che il tuo consenso mi dà un incitamento a rimare [lege: rimanere] ancora «sulla breccia». Con molta difficoltà, giacché gli acciacchi non mancano, ma col coraggio che rende meno insopportabile il tramonto. Qui non posso lavorare se non su cronache ed episodi locali sette-ottocenteschi di cui la Biblioteca e l'Archivio comunale mi offrono la documentazione. Addio Parigi, Roma, Milano, Napoli e le altre grandi biblioteche! Tutt'al più, cerco di aprire, quando posso e sto meglio, i rubinetti vicini dei minori serbatoj di Perugia e di Arezzo...

Ti invidio - ma con ammirazione scevra da ogni impura rivalità - per la tua vicinanza ai grandi depositi bibliografici da cui trai spunto per i tuoi lavori o occasione per le tue ricerche, sempre eccellenti!

Augurando a te, nel tuo nuovo nido, ogni sorta di felicità ed a Francesca il meritato riposo dopo le fatiche del trasloco, ti abbraccio con affetto

Il tuo Raffaele

Poco più di due mesi dopo aver ricevuto questa lettera, il 13 dicembre, assalito da un eccesso di pigrizia, avevo rinunciato ad uscire di casa per andare a prendere il giornale. Stavo così placidamente al mio tavolo di lavoro, quando d'improvviso avverto la 'necessità' di procurarmi il giornale. Una prepotente inattesa energia volitiva mi spinge fuori casa. Eccomi di ritorno con l'abituale quotidiano. Lo sfoglio rapidamente. Arrivo alla pagina degli annunci necrologici (non sempre li guardo) e immediatamente, con sgomento, l'occhio mi si posa sul nome di Raffaele de Cesare. Rimango come incredulo. Sì, è proprio lui, il mio maestro, il mio amico, il mio caro Raffaele: l'annuncio viene dalla sorella Carla, da Città di Castello. Non riesco a fugare la confortante certezza - per quanto la cosa mi possa apparire strana se non addirittura di carattere vagamente mistico - che sia stato lo stesso Raffaele a volermi dare la notizia. Quasi avverto la sua presenza, il calore vivo del suo affetto. Ma con dolore sento anche che il mondo non è più per me quello di prima. 